Background Most accidents occur during leisure time and therefore advanced companies have started to promote leisure time safety at the workplaces. Problem has arisen that employers do not know how much and what kind of leisure time accidents occur to their staff. Therefore, comprehensive leisure time accident information would be important when companies target promoting to right areas of leisure time safety. The aim of this study was to find out effective methods to collect information of leisure time accidents from employees.

Methods Methods for collecting leisure time accident information were contructed and tested in four case companies. In total, five different kind of collecting methods was planned, implemented and validated in cooperation with the case companies.

Results Collecting leisure time accident information was successful with three of the five tested methods. In total, 324 leisure time accident cases were reported by employees with tested methods in four case companies.

Conclusions Experiences of this study suggest that informing and marketing are more important factors in successful collecting of leisure time accident information than actual method to collect. Adding collecting to already existing method rather than creating a totally new one could also improve chances of successful collecting.

\section{DETERMINANTS OF TREATMENT SEEKING BEHAVIOUR FOLLOWING INJURY IN MARINGÁ, BRAZIL}

${ }^{1}$ Nicole Toomey, ${ }^{1,2}$ João Ricardo Vissoci, 1,3Michael Haglund, ${ }^{1,3}$ Catherine Staton. ${ }^{1}$ Duke Global Health Institute; ${ }^{2}$ Faculdade Ingá; ${ }^{3}$ Duke University Medical Centre

\subsection{6/injuryprev-2016-042156.1045}

Background Injury accounts for 6\% of all disability adjusted life years lost. Current research on access to care and injury does not account for injury severity or levels of health care. Our project aims to determine what barriers to injury care arise in Brazil's universal health care system.

Methods Households were randomly selected in Maringá between May 2015 and September 2015. Demographic information was collected for the household; one individual was randomly selected to provide injury history. A chi-square analysis found the association between care-seeking and demographic variables. A preliminary significance level of $\alpha=0.20$ was used for inclusion of variables. A full multivariate logistic regression model and thirty reduced models were run. Mean squared estimate and Akaike Information Criterion were calculated to find the best predictive model.

Results Of the 2678 households and individuals sampled, 30.3\% of individuals reported a lifetime injury. The univariate analysis found that gender $(p=0.034)$, injury cause $(p<0.001)$, race $(\mathrm{p}=0.051)$, severity $(\mathrm{p}=0.103)$ and insurance status $(\mathrm{p}=0.026)$ were predictors of seeking care. Income, and age were included in the multivariate model due to significance in the literature. Based on this model, Burn victims had an increased odds of 7.92 of not seeking care compared to road traffic incident victims; this increased odds was also seen when stratifying by gender $(\mathrm{OR}=9.49$ for women, $\mathrm{OR}=8.23$ for males). For all injuries, being male had a protective effect on seeking care
$(\mathrm{OR}=0.64)$; women had higher odds of not seeking care $(\mathrm{OR}=1.30)$

Conclusions Cause of injury and socioeconomic status factors are a major predictor for seeking care, as are factors related to socioeconomic status. Cause potentially masked the effects of gender on care seeking. More research needs to be done on specific causes of injuries, the role of gender, and why socioeconomic status is still a barrier to care in a universal access system.

\section{THE CANADIAN INJURY PREVENTION TRAINEE NETWORK: BUILDING CAPACITY FOR THE FUTURE OF INJURY PREVENTION RESEARCH}

\begin{abstract}
1,2,35arah A Richmond, ${ }^{1}$ Amanda M Black, ${ }^{2}$ Liraz Fridman, ${ }^{4}$ Allison Ezzat, ${ }^{2}$ Tessa Clemens, ${ }^{5}$ lan Pike, ${ }^{2}$ Alison Macpherson. ${ }^{1}$ Sport Injury Prevention Research Centre, Faculty of Kinesiology, University of Calgary, Canada; ${ }^{2}$ Faculty of Kinesiology and Health Sciences, York University, Canada; ${ }^{3}$ Child Health Evaluative Sciences, Hospital for Sick Children, Canada; ${ }^{4}$ School of Population and Public Health, University of British Columbia, Canada; ${ }^{5}$ Department of Paediatrics, University of British Columbia and BC Injury Research and Prevention Unit, Child and Family Research Institute, Canada
\end{abstract}

\subsection{6/injuryprev-2016-042156.1046}

Background Injuries are a serious but preventable health concern in Canada and a growing field of research, attracting a large number of graduate students and other trainees across Canada to identify injury prevention as their field of study. In 2009, the Canadian Institute for Health Research funded a team in Child and Youth (C\&Y) Injury Prevention. With this support, the team was able to develop a model of practice involving researchers, stakeholders, knowledge users, and trainees as part of a multidisciplinary approach to reducing the burden of injury in youth. The C\&Y team was successful at supporting and highlighting the work of over 40 team trainees. The efforts of this trainee team have resulted in the formation of the Canadian Injury Prevention Trainee Network (CIPTN).

Objective The CIPTN aims to build a network of trainees interested in the science and practice of injury prevention (IP) from a multi-disciplinary perspective. Specifically, the goals include increasing opportunities for collaboration, professional development, mentorship and networking.

Results The CIPTN has worked to develop a list of learning and research oriented catalyst activities for trainee members. The CIPTN has successfully collaborated with IP experts and organisations from across Canada in the development of a comprehensive IP resource, as well as updating the Canadian Injury Prevention Curriculum, a course for IP practitioners across Canada. Further, an executive board and governance structure has facilitated the identification of future collaborative activities (e.g., bi-monthly seminars, grant writing, and evaluating an injury methods workshop).

Conclusions The CIPTN increases opportunities for junior researchers and trainees to work within a network of colleagues to share research, build collaborative projects, expand capacity, and provide training opportunities. This in turn, will develop the quantity and quality of IP science and practice across Canada for future generations. 